\title{
DEVELOPMENT OF LONGITUDINAL COUPLING IMPEDANCE MEASUREMENT PLATFORM FOR BEPCII *
}

\author{
Gang Huang, Huang Wenhui, Shuxin Zheng (Tsinghua University, Beijing), \\ Demin Zhou, Jiuqing Wang(IHEP Beijing, Beijing)
}

\begin{abstract}
A coaxial line impedance measurement platform is developed for BEPCII under the collaboration between Tsinghua University and IHEP. A pair of gradual change impedance matching section is designed and fabricated by numerical control milling machine. The special designed $\mathrm{RF}$ connector is applied to strengthen the inner conductor. The algorithm of TRL calibration is applied in the system to avoid the usage of a reference pipe for each device under test. The measurement is accomplished by a VNA under the control of the software written in LabView.
\end{abstract}

\section{INTRODUCTION}

Longitudinal coupling impedance is one of the important reasons for Multibunch instability in storage ring ${ }^{[1,2,3]}$. Measure the longitudinal impedance of vacuum components is helpful for optimization and makes it possible to analysis the performance of the storage ring later. Coaxial line impedance measurement is a standard bench impedance measurement method.

A coaxial line longitudinal impedance measurement platform for BEPCII is developed by Tsinghua University and distributed to IHEP by May 2004, as shown in figure $1 .^{[4,5]}$.

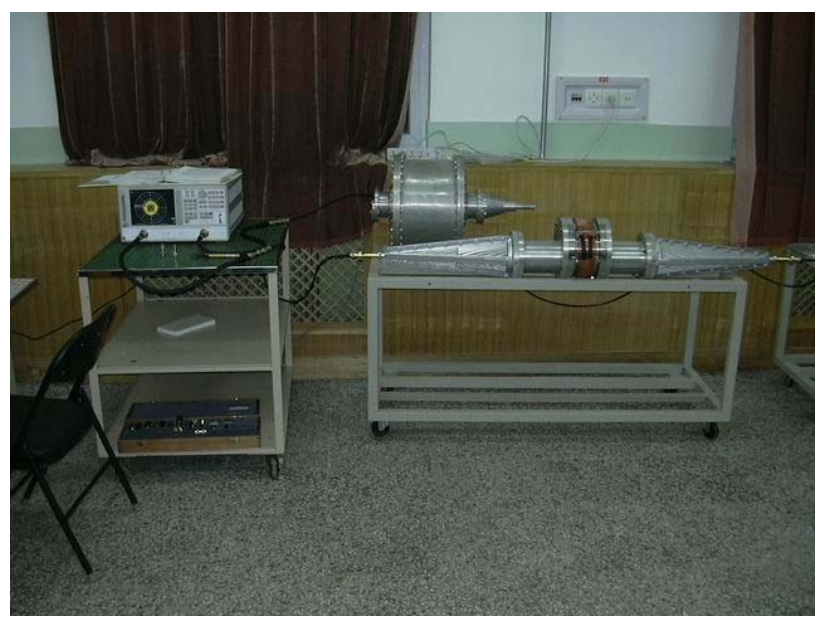

Figure 1: Longitudinal coupling impedance measurement platform for BEPCII.

The coaxial line longitudinal impedance measurement platform contains a pair of gradual change impedance matching section, a pair of special designed RF connector, the control and user interface software and other supporting parts. TRL calibration is applied in the platform to correct the reflection and insertion loss of the

*Work supported by NSFC 10375035 .

"huanggang@tsinghua.edu.cn
RF transmission system. The part performances are measured by vector network analyzer (VNA). The impedance of a pillbox cavity is measured and compared with the simulation of MAFIA.

\section{COAXIAL LINE IMPEDANCE MEASUREMENT PLATFORM}

Coaxial line impedance measurement places a wire in the center of the Device Under Test (DUT) and forming a coaxial line with the DUT. A current pulse is propagated on the coaxial line. The field excited by the pulse is similar to the field excited by a short pulse. The response of the current pulse contains the impedance information of the DUT. This measurement can be carried out either in time domain or in frequency domain. In frequency domain, the measurement is degenerated into the measurement of the $\mathrm{S}$ parameter of the coaxial lines. Such measurements are implemented by VNA.

\section{Gradual change impedance matching section}

The cross-section of the DUT is octagonal and the connector of the VNA used is N-type. A matching section is required to convert the octagonal cross-section into circle cross-section. Gradual change impedance matching section is selected to minimize the RF reflection in a limited space. Since every cross-section in such a matching section is not simple shape, a numerical milling

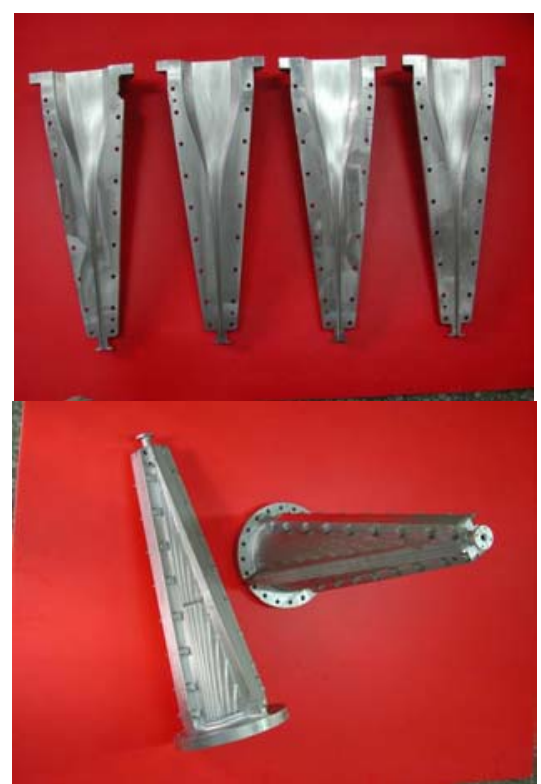

Figure 2: Gradual change impedance matching sections. Upper for inside face and lower for assembled parts. 
machine is applied to fabricate it. The matching section is fabricated by half and assembled by dowel. The fabricated gradual change impedance matching section is shown in figure 2 .

The inside face of the section is a little complex. The generator of it is designed as double cosine function. Since the lowest mode in the coaxial line is TEM mode, the image current on the outer conductor has only longitudinal part. The transfer function of the coaxial line can be derived by idea of infinite thin filters parallel. The equation below shows the impedance of each slice of the generator and the relative reflection factor.

$$
\begin{gathered}
Z_{\text {double } \cos }(z)=\sqrt{Z_{1} Z_{2}} e^{\left(\frac{z}{l}-\frac{1}{2}\right) \ln \frac{Z_{2}}{Z_{1}}}\left(1+\frac{\sin \left(\frac{2 z \pi}{l}-\pi\right)}{\frac{2 z \pi}{l}-\pi}\right) \\
\left|\Gamma_{\text {double } \cos , 1}\left(\frac{1}{\lambda}\right)\right|=\left|\frac{1}{2} \ln \frac{Z_{2}}{Z_{1}} \frac{\pi^{2}}{\pi^{2}-\left(\frac{2 \pi l}{\lambda}\right)^{2}} \frac{\sin \frac{2 \pi l}{\lambda}}{\frac{2 \pi l}{\lambda}}\right|
\end{gathered}
$$

The reflection of such a parallel filter is still very small when the reflection of each generator is small.

The performance of the gradual change impedance matching section is measured in pair by VNA and then derived to single. The measured reflection is shown in figure 3 . The reflection of the section is less then 0.2 in a frequency range below $8 \mathrm{GHz}$.

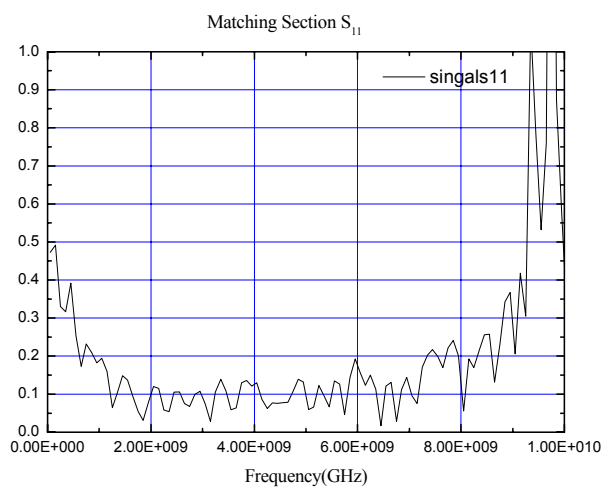

Figure 3: performance of the gradual change impedance matching section

\section{Special designed rf connector}

The measurement platform requires the inner connector to be located at the center of the DUT and strengthened enough to imitate the behavior of electron beam. The impedance measurement requires more than one measurement to obtain the impedance, that require the connector have good repeatability.

A special designed RF connector is applied to meet the requirement of repeatability, and capability to strengthen the inner conductor. ${ }^{[6]}$ The electric boundary doesn't change when the inner conductor is being strengthened. The drawing of the connector is shown in figure 4 . The left side is a standard N-type connector and the right side connected to the relocated inner conductor. The performance of the conductor is measured in pair by VNA, And the single connector $\mathrm{S}_{21}$ is calculated and shown in figure 5. The reflection of the connector is less then 0.2 in a frequency range below $13 \mathrm{GHz}$.

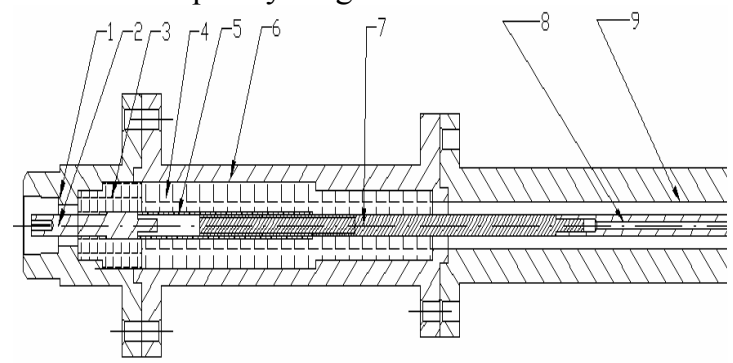

Figure 4: Special designed RF conductor

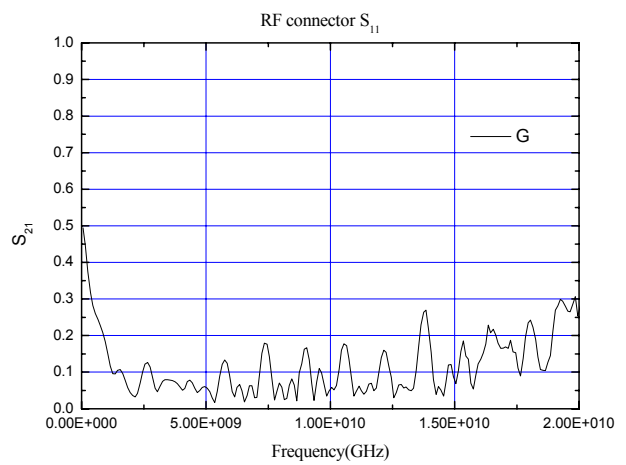

Figure 5: $\mathrm{S}_{21}$ of the special designed RF connector

\section{TRL CALIBRATION}

Typically the coaxial line impedance measurement platform precedes a measurement on the Device Under Test (DUT) together with a measurement on the Reference pipe (REF). The reference pipe should have the same length with the DUT. A TRL calibration is applied in this platform to eliminate the usage of a reference pipe. ${ }^{[7]}$ The block diagram of the measurement is shown in figure 6. Four standard calibrate kit measurements are performed to correct the reflection of the RF transmission system.

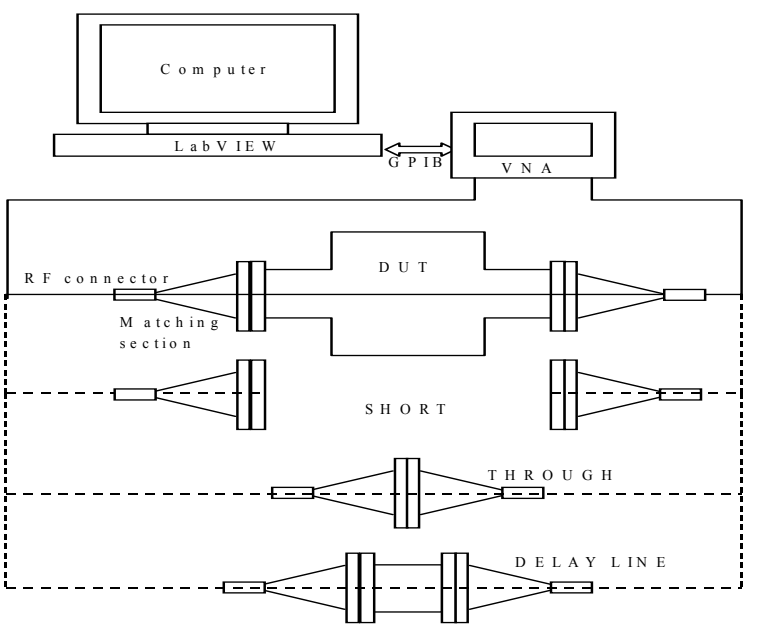

Figure 6: TRL based longitudinal impedance measurement 


\section{SOFTWARE}

Computer control measurement software is written in LabVIEW and distributed to IHEP. Figure 7 is the user interface of the software. Following the highlighted control, user can process the whole measurement including the TRL calibration. The software through GPIB interface controls the vector network analyzer.

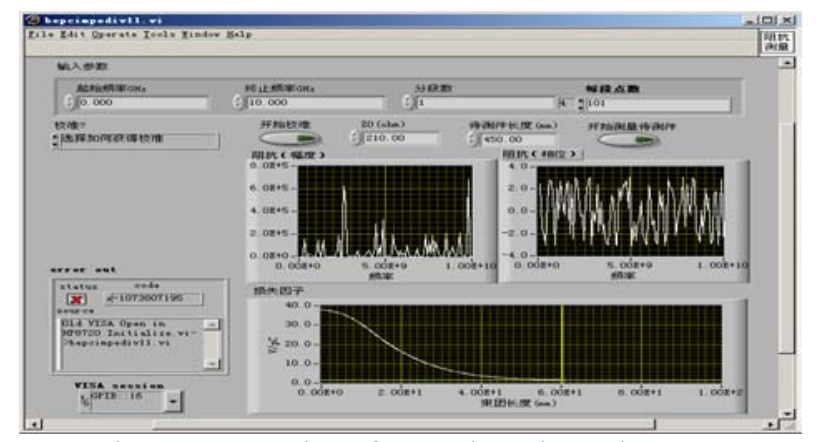

Figure 7: User interface written by LabVIEW

\section{RESULT}

To verify the availability of the longitudinal impedance measurement platform, a pillbox cavity is measured. The measured $S_{21}$ and the calculated result by MAFIA are compared by figure 8 . The position and the amplitude of the peaks are agreed quite well.
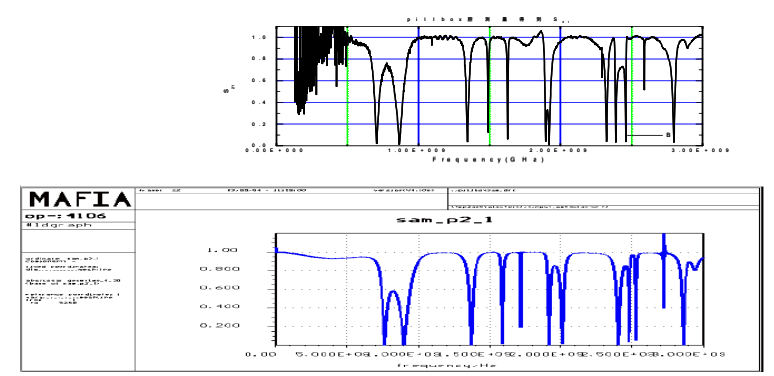

Figure 8: Measured and calculated S21 of the pillbox

\section{CONCLUSION AND IMPROVEMENT}

A longitudinal coupling impedance measurement platform for BEPCII is developed by Tsinghua University and delivered to IHEP on May 2004. The gradual change impedance matching section and the special RF connector are designed and fabricated. The performance of them met the requirement or BEPCII. TRL calibration is applied in the platform and the computer-controlled software is provided.

The precision control of gradual change impedance matching section is not good enough that the small port have some ripple. The cables used in the platform are not good enough that have phase and insertion loss change between different measurements.

According to the users' report: 'the impedance, larger than $20 \mathrm{Ohm}$ and below $6 \mathrm{GHz}$, can be measured using the TRL calibration technique in the experiment. ${ }^{[5]}$

\section{REFERENCES}

[1] M. Sands and J. Rees, "A Bench Measurement of the Energy loss of a stored beam to a cavity," PEP-95. 1974

[2] G. Huang, "Longitudinal Broadband Impedance Measurement System by Coaxial Line Methods," PAC'01, Chicago, June 2001,p2060

[3] G. Huang, W. Huang et., "The Study of Longitudinal Coupling Impedance Measurement and Beam Feedback System" PhD thesis, Tsinghua University, 2002

[4] G. Huang and S. Zheng et., "Updating the Longitudinal Coupling Impedance Measurement Platform for BEPC," APAC2004, Gyeongyu, Korea, March 2004

[5] D. Zhou, and W. Kang et., "Longitudinal Impedance Measurements of the Components for the BEPCII" this proceeding

[6] G. Huang and W. Huang et., "Developing a Special RF Connector for Coaxial Impedance Measurement Platform,". High Energy Physics and Nuclear Physics. Vol. 26, No. 5, 2002 p92

[7] G. Huang and W. Huang et., "Application of TRL Calibration in Longitudinal Coupling Impedance Measurement Platform for BEPCII" this proceeding. 